Linguistique, littérature, didactique

167-168 | 2015

L'exception (revue et corrigée)

\title{
Faute avouée à moitié pardonnée
}

Les figures du discours : des fautes pardonnables?

A Fault Confessed is half Redressed. Are Figures of Speech Forgivable Mistakes?

\section{Geneviève Salvan}

\section{OpenEdition}

Journals

Édition électronique

URL : http://journals.openedition.org/pratiques/2712

DOI : $10.4000 /$ pratiques. 2712

ISSN : 2425-2042

Éditeur

Centre de recherche sur les médiations (CREM)

Référence électronique

Geneviève Salvan, « Faute avouée à moitié pardonnée », Pratiques [En ligne], 167-168 | 2015, mis en ligne le 04 avril 2016, consulté le 21 avril 2019. URL : http://journals.openedition.org/pratiques/2712 ; DOI : $10.4000 /$ pratiques. 2712

Ce document a été généré automatiquement le 21 avril 2019

(c) Tous droits réservés 


\section{Faute avouée à moitié pardonnée}

Les figures du discours : des fautes pardonnables?

A Fault Confessed is half Redressed. Are Figures of Speech Forgivable Mistakes?

Geneviève Salvan

\section{Introduction : la relativité des jugements de la figure comme faute}

1 Avec une réticence toute rhétorique, Georges Molinié (1986: 103) évoque ainsi l'anacoluthe: "On ose à peine évoquer l'anacoluthe, traditionnellement expliquée comme une rupture de construction », avant d'ajouter malicieusement : "c'est pourtant bizarre, de donner un nom de figure à ce que tout l'enseignement du français appelle une faute. Ou plutôt, c'est intéressant». Le «scandale» intralinguistique que constituent certaines figures a longtemps conduit à les exclure de la linguistique et à les traiter en termes d'écart ou de faute, quand ce n'est pas de vice.

2 Or, les figures sont bel et bien des « réalités langagières incontournables " (Bonhomme, 2013 : 3) et le regain d'intérêt de la linguistique contemporaine pour elles l'atteste. Elles sont prises entre grammaire et rhétorique, relevant de la grammaire en ce qu'elles affectent la structure formelle et sémantique des énoncés et de la rhétorique en ce qu'elles génèrent des marquages discursifs donnant lieu à des effets stylistiques. La remarque de G. Molinié souligne cette tension dans une figure telle que l'anacoluthe qui intègre, dans sa définition même (rupture de construction), l'entorse à la règle syntaxique. C'est cette position entre grammaire et rhétorique qui fonde la dialectique de la figure et de la faute, rhéteurs et grammairiens se renvoyant dos à dos les figures comme leurs objets propres.

3 La figure est l'objet de plusieurs types de jugement: celui qui la tolère ou la valorise comme ornement (elle est belle, appropriée mais décorative), celui qui la catégorise comme écart (elle déroge à l'usage) et, enfin, celui qui la condamne comme faute (elle déroge à la langue). Plusieurs termes sont employés pour (dis)qualifier l'inconduite des 
figures : outre la faute (dite parfois d'écriture), il y a le défaut (en général de style), le vice ( d'élocution) ou encore l'entorse (à la règle). Cette dialectique de la figure et de la faute s'ancre dans l'insertion progressive des figures dans la grammaire au Moyen Âge et à la Renaissance, « insertion qui se prolongera chez les grammairiens philosophes des siècles suivants " (Gardes Tamine, 2011: 62), notamment sous la plume de Dumarsais dans l' Encyclopédie. Cette intégration place de fait certaines figures aux marges de la grammaticalité : plus le discours grammatical est fort et normatif, plus les figures risquent d'être perçues comme des trublions et reversées soit dans les faits de parole (singuliers, et parfois à traiter comme hapax), soit dans les fautes.

4 En fait, le jugement de la figure comme faute doit être triplement relativisé :

- d'un point de vue historique :

- 1) la figure dépend de l'état de langue dans laquelle on l'envisage. L'hyperbate (Albe le veut, et Rome, Corneille), figure de perturbation syntaxique, a vu sa définition notoirement changer depuis les Grecs et les Latins, qui insistent dans le cadre de leur langue sur la perturbation par inversion, jusqu'au français où les théorisations de la figure l'emmènent plutôt vers l'idée d'une rallonge inattendue de la phrase (Stolz, 2011). L'hyperbate travaille donc la norme de manière différente, qu'elle exploite la laxité de l'ordre des mots ou qu'elle en transgresse la fixité ;

- 2) Si la figure dépend de l'état de langue et de la norme qui y est attachée, elle est aussi tributaire de l'idéologie linguistique dominante et du sentiment linguistique modelé par celle-ci. Ainsi, l'anacoluthe (Et pleurés du vieillard, il grava sur leur marbre / Ce que je viens de raconter, La Fontaine) joue avec les règles de bonne formation de la phrase, dont on sait qu'elle est une des notions centrales dans l'imaginaire de la langue. L'anacoluthe ne sera perçue comme «dystaxie » qu'à partir du moment où s'impose la norme de la «netteté mécanique des rapports » syntaxiques. Fortement dévalorisée, elle prête alors son nom aux grammairiens qui en "affublent tout ce qui n'est pas mathématiquement conséquent » (Seguin, 1999 : 333);

- d'un point de vue stylistique : à partir du moment où la figure prête sa saillance ${ }^{1}$ à un marquage expressif, remarquable et révélateur d'une "tension singularisante» (Bonhomme, 2008: 1491), la prétendue faute sur laquelle elle repose est résolue par sa motivation stylistique. Ainsi l'oxymore (Cette obscure clarté qui tombe des étoiles, Corneille), une fois dépassé l'acte de prédication non pertinent et la «violation apparente de la logique » (Fromilhague, 1995: 54), peut-il libérer son potentiel figural dans un texte qui développe par exemple la vision d'une réalité à double fond, pour la dénoncer ou en montrer la complexité ;

- d'un point de vue linguistique enfin: il n'y a pas de coupure épistémologique entre grammaire et stylistique, mais un continuum entre « les structures générales de la langue et les procédés particuliers d'expression » (Bonhomme, 2000 : 11). Autrement dit, la figure ne se construit pas en marge de la langue, mais elle tire parti de ses potentialités, en exploite les zones non entièrement codées, et la figuralité nait « dès qu'une virtualité de la langue échappe aux contraintes normatives pour donner lieu à un marquage discursif idiolectal » ( ibid.).

5 Les figures, malgré leur composante rhétorique forte, sont donc souvent l'objet de discours grammaticaux normatifs et de jugements axiologiques qui opèrent comme des filtres : ce sont ces jugements d'exclusion, et les stratégies de réintégration dans l'analyse linguistique actuelle, qui nous intéresseront ici. Dans chacune des deux parties, consacrées à une figure en particulier, seront examinés (1) un certain type de jugement 
négatif et son rapport à la norme sous-jacente et (2) l'apport de l'analyse linguistique et stylistique dans la réévaluation de la figure. Nous avons retenu deux figures, le pléonasme et l'hypallage, l'une plus connue que l'autre, qui ont une définition et une réception ambigües du fait de leur positionnement problématique entre grammaire et rhétorique et de leur lien avec la pratique rédactionnelle normative.

\section{Le pléonasme, aux marges ou aux marches de la figuralité ?}

6 Les jugements dévalorisants sont à l'origine de l'éviction pure et simple de certaines figures quand elles dérogent à un possible de la langue, ou de leur marginalisation, quand on les cite sans leur reconnaitre le statut de figure. Ce type de jugement est typique de la manière d'appréhender le pléonasme, figure injustement mal aimée, et régulièrement bannie de la figuralité.

\subsection{Le pléonasme en procès}

\subsubsection{La redondance indue}

7 Lorsqu'il n'est pas purement et simplement évincé des traités, le pléonasme fait l'objet de jugements ambivalents, dépréciatifs ou valorisants, excluants ou récupérateurs. Classé dans les figures de construction par redondance, il est généralement condamné pour deux défauts majeurs, relevant (1) d'un possible de la langue et (2) de la conduite des échanges verbaux: (1) la redondance sémantique, qui fait de lui une «faute de logique verbale » (Robrieux, $2010: 148)$; et (2) la superfluité discursive, infraction à la maxime de quantité (Grice, 1979), qui veut qu'un énoncé donne un nombre restreint mais juste d'informations. Les définitions actuelles s'appuient toutes sur une longue tradition rhétorique que Beauzée résume ainsi dans son article «Pléonasme » de l'Encyclopédie, en citant Dumarsais :

(1) Il y a pléonasme, dit M. du Marsais, article figure, lorsqu'il y a dans la phrase quelque mot superflu, ensorte que le sens n'en seroit pas moins entendu, quand ce mot ne seroit pas exprimé ; comme quand on dit, je l'ai vù de mes yeux, je l'ai entendu de mes oreilles, j'irai moi-même; mes yeux, mes oreilles, moi-même sont autant de pléonasmes.

Bien avant lui, les rhéteurs de l'Antiquité et de la Renaissance dénonçaient déjà le pléonasme comme "solécisme par addition" (Quintilien, Institution oratoire) et « superfluyté du langaige » (Fabri, Le Grand et vrai art de pleine rhétorique). Privilégiant un sens étymologique au détriment de l'autre, ces définitions sont partielles et partiales : le

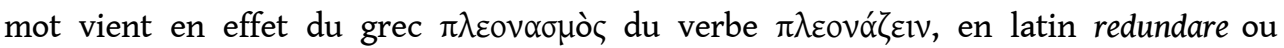
abundare. Il signifie aussi bien abondant, et même surabondant, excessif, que redondant, et donc superflu, ce qui conduit Beauzée à préciser que «le mot de pléonasme signifie ou plénitude ou superfluité ». De ces deux sens antiorientés, le discours rhétorique préfèrera celui de "superfluité » et oubliera le sens d'«abondance ». Le pléonasme devient alors le parangon de la répétition vicieuse, « défaut du style, ou du moins [une] négligence qu'on doit éviter » (Dumarsais), parce qu'inutile et «mal appropriée » au discours, c'est-à-dire impertinente. Les jugements des dictionnaires sont ainsi sans appel, à l'instar du 
Dictionnaire de l'Académie dans ses éditions de 1694 et de 1762, qui choisit pourtant le terme abondance dans sa définition :

(2) Abondance de paroles qui ne signifient que la mesme chose. Le pleonasme est presque tousjours vicieux dans le discours.

9 Ce n'est qu'à partir de l'édition de 1798 que le Dictionnaire de l'Académie l'évoque comme figure, lui concédant un effet discursif d'expressivité - repris des Encyclopédistes Dumarsais et Beauzée -, que démentent toutefois encore l'exemple et la remarque finale :

(3) Figure par laquelle on ajoute des mots inutiles pour le sens d'une phrase, mais qui peuvent y mettre de la force ou de la grâce. Quand les mots n'ajoutent rien, le pléonasme est vicieux.

Pléonasme ne se prend plus guère qu'en mauvaise part, et signifie, Une rédondance vicieuse de paroles.

10 Les dictionnaires de langue actuels poursuivent l'assimilation de la faute contre la langue et de l'impertinence du discours, quelle que soit l'intentionnalité qui y préside, tout en soulignant que certains pléonasmes sont devenus des lieux communs et ne gênent pas la communication :

(4) Terme ou expression qui ajoute une répétition (consciente ou inconsciente) à ce qui a été énoncé. Synon. battologie, périssologie, tautologie. Les expressions « je l'ai vu de mes yeux, entendu de mes oreilles » sont des pléonasmes admis et fort usités. (TLFi)

11 Entre fait grammatical et fait rhétorique, le pléonasme a du mal à trouver sa place.

\subsubsection{Il y a cependant pléonasme et pléonasme : la périssologie}

12 Une première stratégie de réintégration du pléonasme dans la figuralité tient à un souci de linguiste. Fort du constat que «c'est un défaut dans le langage grammatical de désigner par un seul \& même mot deux idées aussi opposées que le sont celle d'une figure de construction \& celle d'un vice d'élocution », Beauzée distribue sur deux termes disponibles ce qui relève de la « couleur de rhétorique » (Fabri) et ce qui relève de la faute linguistique : à la figure le pléonasme, au défaut la périssologie.

13 Malgré l'apparent confort de cette distinction, la répartition des rôles dans le doublet figure/faute n'est cependant pas aussi claire. D'une part, les exemples choisis pour illustrer les deux figures sont souvent les mêmes (l'exemple prototypique étant j'ai vu de mes yeux vu), donnant au seul contexte de l'exemple la responsabilité de la figure ou de la faute; d'autre part, le terme périssologie n'est pas utilisé dans le langage courant. Enfin, le pléonasme, se fondant de toute manière sur la redondance, est toujours suspect et inconditionnellement condamné par les puristes. Le sentiment linguistique contre la surenchère sémantique est très fort, en témoignent aussi bien la réaction pleine d'humour d'Arletty lorsqu'elle déclare que «fermer les maisons closes, c'est plus qu'un crime, c'est un pléonasme " ou celle plus hargneuse - et plus suspecte - d'un puriste anonyme qui fustige sur son blog " cet horrible "au jour d'aujourd'hui" » qui « double le pléonasme régulier d'un pléonasme fautif $»^{2}$. Le pléonasme n'est « régulier » que par son ancienneté et la caution de la lexicalisation... 


\subsection{La figuralité reconnue mais restreinte à l'expressivité}

14 Ce sont alors les conditions du discours qui volent au secours du pléonasme, justifié par des motivations singulières, en général qualifiées de "stylistiques ». La faute contre la langue est excusée par les nécessités d'une communication complexe, typique du discours littéraire, dans laquelle l'autorité du locuteur légitime le choix de la redondance. L'indulgence des critiques est alors souvent étayée par un jugement d'expressivité, comme l'illustre ce commentaire d'Arlette Éboué, cité et repris à son compte par N. Ricalens-Pourchot (2010: 102), à propos d'un pléonasme chez Baudelaire :

(5) « (...) quand Baudelaire écrit :

Envole-toi bien loin de ces miasmes morbides... (Baudelaire, Les Fleurs du Mal, «Élévation»)

il souligne son dégoût du monde par un pléonasme dont l'expressivité est renforcée par l'allitération du $m$ »(p. 803).

Le pléonasme n'est une figure que s'il est employé pour donner plus d'énergie et de clarté à l'expression de la pensée ou même parfois pour créer des effets comiques. Les grands auteurs eux-mêmes n'en dédaignent pas l'utilisation.

Le jugement en termes d'expressivité distingue alors pléonasme vicieux et littéraire, et correspond à l'exact inverse de la stratégie précédente qui consistait à distinguer par deux mots ce qui est faute et figure. Il s'agit dans ce cadre d'assimiler par le même mot deux réalités discursives pourtant bien distinctes. Le pléonasme est alors évalué à la seule lumière d'une intentionnalité et d'une visée à la fois discursive et esthétique, qui empêche qu'on ne le pense comme véritable schème figural, à la figuralité variable.

\subsection{En fait, le vrai pléonasme se moque du pléonasme}

Plutôt que de distinguer par des mots différents les pléonasmes défectueux et appropriés, on a tout intérêt à envisager une gradualité du régime de la figuralité du pléonasme en fonction des conditions du discours, la redondance sémantique produisant plus ou moins " un relief jugé exemplaire, et par là figural, au contact du discours et de ses récepteurs » (Bonhomme, 2009: 122). Il s'agit alors de ne réserver le terme de pléonasme qu'aux réalisations figurales de la redondance. Mais en quoi consiste la figuralité du pléonasme?

Lorsqu'il est reconnu comme figure, le pléonasme est défini comme «variété de caractérisation non pertinente exactement inverse de l'oxymore» (Molinié, 1992: 272), affectant un mot d'un caractérisant sémantiquement incongru parce que redondant ${ }^{3}$. La définition de Beauzée, dont on n'a retenu que la force expressive, précisait déjà le rendement figural du pléonasme :

(6) Il y a pléonasme lorsque des mots qui paroissent superflus par rapport à l'intégrité du sens grammatical, servent pourtant à y ajouter des idées accessoires, surabondantes, qui y jettent de la clarté ou qui en augmentent l'énergie. (article Pléonasme de l'Encyclopédie)

Autrement dit, le pléonasme opère en discours un élargissement sémantique ${ }^{4}$ et traduit un investissement énonciatif. Si le pléonasme n'apporte rien au «sens grammatical », il introduit un "dire en plus » en activant des « idées accessoires » qui peuvent avoir une valeur ampliative (et soutenir par exemple une hyperbole), désambiguïsante (être clair) 
ou expressive (augmenter l'énergie). La redondance, réévaluée comme activation de "connotations additionnelles par rapport au contenu encyclopédique transmis" (Bonhomme, 2005a : 118-119), prend en discours une visée pragmatique, qui oblige «non seulement de prendre ensemble le locuteur, le récepteur, le message et le référent, mais de modéliser le processus même de leur interaction » (Verine, $2008: 120)$.

La réévaluation du pléonasme passe donc par la prise en compte des conditions du discours et de l'énonciateur : ce dernier, en choisissant la figure dit exactement ce qu'il veut dire et propose son expression comme la plus juste en contexte. Ce faisant, il se positionne par rapport au réel perçu/vécu et par rapport à l'autre, qu'il s'agisse d'autrui ou de lui-même (Gaudin-Bordes \& Salvan, 2009 : 121). Le pléonasme, même lorsqu'il est considéré comme une simple maladresse d'expression, joue avec la caractérisation attendue et discute les découpages notionnels conventionnels en y inscrivant l'énonciateur et le récepteur: par exemple, qu'est-ce qui motive le choix en discours d'un "soleil jaune »?; qu'est-ce que fait un "soleil jaune " sur la représentation du soleil?

Soit le sonnet de Rimbaud «Le Buffet», paru dans le recueil Poésies en 1870, dans lequel on lit un pléonasme d'autant plus saillant qu'il s'accompagne d'une antéposition marquée de l'adjectif et qu'il repose sur une parenté morphologique entre le caractérisé et le caractérisant $^{5}$ :

(6) C'est un large buffet sculpté, le chêne sombre,

Très vieux, a pris cet air si bon des vieilles gens;

Le buffet est ouvert, et verse dans son ombre

Comme un flot de vin vieux, des parfums engageants;

Tout plein, c'est un fouillis de vieilles vieilleries,

De linges odorants et jaunes, de chiffons

De femmes ou d'enfants, de dentelles flétries,

De fichus de grand-mère où sont peints des griffons ;

- C'est là qu'on trouverait les médaillons, les mèches

De cheveux blancs ou blonds, les portraits, les fleurs sèches

Dont le parfum se mêle à des parfums de fruits.

- ô buffet du vieux temps, tu sais bien des histoires,

Et tu voudrais conter tes contes, et tu bruis

Quand s'ouvrent lentement tes grandes portes noires.

21 Pour évoquer le meuble familial, ce «fouillis de vieilles vieilleries », Rimbaud fait le choix surprenant $\mathrm{du}$ pléonasme. Dans l'économie du poème, celui-ci relaie néanmoins efficacement l'accumulation valorisante des objets composant le buffet, avec lesquels il fait corps (le buffet est d'ailleurs personnifié dans le second tercet grâce à l'apostrophe).

L'adjectif pléonastique asserte une propriété conventionnellement attachée au référent de « vieilleries » (et définitoirement attachée au sens du nom), et agit apparemment peu sur la représentation de ce référent, contrairement à ce que ferait l'adjectif oxymorique jeune dans une jeune vieillerie, qui bousculerait notre représentation du référent. Le propre d'une qualification pléonastique est de redire l'une des propriétés du nom et de coller à la représentation conventionnelle du référent. Si la caractérisation parait tout d'un coup incongrue, c'est parce qu'elle est tellement appropriée qu'elle en devient suspecte. Le pléonaste $^{6}$, loin de se distinguer en proposant une vision contradictoire, polémique ou seulement singulière, fait mine d'appréhender la réalité dans une situation donnée de manière conventionnelle, et surtout linguistiquement préconstruite. Se rangeant avec zèle à la doxa, il colle à la définition commune de la notion au risque de se voir sanctionné par l'interlocuteur («une vieillerie est toujours/forcément vieille!»). De quoi le 
pléonasme tire-t-il alors son efficace, et en quoi peut-il constituer une figure pertinente et fonctionnelle?

Le pléonasme oriente le domaine des vieilleries vers ce qu'A. Culioli appelle l'attracteur, «occurrence idéale de la notion, inatteignable et indicible» (Mellet, 2012: 13), et constitue une tentative en discours d'exprimer cet attracteur7. Il crée simultanément une zone frontière délimitant, d'une part, les vieilleries et, d'autre part, ce qui n'est pas des vieilleries, zone dans laquelle on trouve des vieilleries pas vraiment vieilles. Il introduit également un gradient qui permet de saisir, dans le domaine des vieilleries, certaines plus ou moins vieilles. La « vieille vieillerie » n'est pas une occurrence normale, mais une qui se singularise par l'intensité de sa propriété définitoire. La prise en charge énonciative d'une référence singulière n'est donc pas niée, mais bien affirmée et tirée vers l'attracteur. C'est pourquoi le pléonasme prend souvent en contexte une dimension valorisante, voire hyperbolique qui le justifie énonciativement. Il n'a d'ailleurs besoin d'aucun discours d'escorte, ce qui le distingue de la tautologie qui assimile l'occurrence envisagée non à l'attracteur mais au type ${ }^{8}$ et qui enferme la notion dans la simple désignation, comme dans « une femme est une femme, il n'y a pas à dire ».

Dans l'exemple de Rimbaud, le pléonasme est proche de l'épithète de nature (la blanche colombe), mais celle-ci se contente pour sa part de dire une occurrence typique et clichéique, en renvoyant à des « qualités codées dans la culture, un univers où l'objet ne s'appréhende que comme l'incarnation d'un stéréotype où sont sédimentées un certain nombre de valeurs " (Maingueneau, 1990 : 125). Le pléonasme oriente quant à lui vers l'attracteur dans une logique "évaluative " de l'occurrence qui le rend propre à l'hyperbole (négative ou positive), ce que ne fait pas l'épithète de nature qui se contente de construire un type. En outre, le pléonasme ne se positionne pas par rapport au cliché ou au stéréotype; il acquiert sa figuralité - instable et négociée dans les échanges verbaux dans lesquels il apparait - de sa fonctionnalisation dans le discours, puisqu'il traduit un investissement énonciatif (valoriser une occurrence) et un gain interprétatif (construire une frontière notionnelle là où il n'y en avait pas) ${ }^{9}$.

On ne s'étonnera pas que certains aient pu qualifier le pléonasme de Rimbaud de «faux pléonasme »: les préjugés ont en effet la vie dure... et, dans cette doxa, seule la récupération par le genre du discours, l'autorité de celui qui parle ou l'efficacité de la formule, rendent incontestable discursivement une figure qui continue pour les puristes à être contestable linguistiquement.

\section{La fausseté des figures démasquée : l'hypallage}

\subsection{La figure comme falsification d'un fait linguistique...}

Une autre stratégie d'exclusion est celle qui consiste à critiquer la fausseté des figures. À la figure faute, qui pèche par manquement aux règles linguistiques, on substitue la figure fausse, qui procède par falsification et dénaturation d'un fait linguistique en le rendant contraire à ce qu'il doit être. Là où le discours normatif censurait, ce discours plus descriptif dévoile la supercherie et rabat la figure sur un emploi détourné d'un fait linguistique. Ainsi l'interrogation rhétorique (ex. 8) est-elle souvent définie comme une fausse question (Ricalens-Pourchot, 2010 : 74) ou une pseudoquestion (Dupriez, 1984), la subjection (ex. 9) comme un faux dialogue parce qu'il consiste à "présenter une affirmation sous la forme question-réponse, dans un simulacre de dialogue » (Robrieux, 
2010 : 124), le dialogisme (ex. 10) est quant à lui un « questionnement fictif » (ibid. : 125). L' attelage (ex.11), parce qu'il met sur le même plan fonctionnel deux éléments dissemblables (Fromilhague, 1995 : 41), devient une «fausse coordination » (RicalensPourchot, 2010 : 38). Enfin, l'hypallage (ex. 12), par sa caractérisation oblique, est parfois désignée comme « fausse attribution » (Cotte, $1991: 80)$ :

(7) Dom Pourceau raisonnait en subtil personnage :

Mais que lui servait-il ? Quand le mal est certain,

La plainte ni la peur ne changent le destin.

(La Fontaine, "Le cochon, la chèvre et le mouton ", cité in: Bonhomme,

$2012: 39-40)$

(8) Elvire, où sommes-nous? et qu'est-ce que je voi?

Rodrigue en ma maison! Rodrigue devant moi !

(Corneille, Le Cid, cité in: Dupriez, 1984 :372)

(9) L'ambition, l'amour, l'avarice, la haine

Tiennent comme un forçat son esprit à la chaîne.

Le sommeil sur ses yeux commence à s'épancher :

Debout, dit l'avarice, il est temps de marcher.

- Hé ! laissez-moi. - Debout ! - Un moment. - Tu répliques?

- À peine le soleil fait ouvrir les boutiques.

- N'importe, lève-toi. - Pour quoi faire, après tout?

- Pour courir l'océan de l'un à l'autre bout.

(Boileau, Satires, VIII, cité in: Robrieux, 2010 : 125)

(10) Quelque part entre la ville et la forêt, des tirs de mitrailleuses commencent à se faire entendre, indices d'échanges sérieux et que l'armée soviétique pourrait vraiment n'être pas loin. (J. Echenoz, Courir, Paris, Minuit, 2008, p. 27)

(11) Le bruit plat et liquide des avirons et la brume lunaire creusaient encore le silence de peste (Gracq, Le Rivage des Syrtes, Paris, Corti, 1951, p. 83)

Les différents emplois du qualificatif fausse masquent des nuances sémantiques: une interrogation rhétorique est fausse parce qu'elle se donne pour ce qu'elle n'est pas, de même la subjection et le dialogisme sont des simulacres parce qu'ils ont l'apparence trompeuse d'un dialogue, tandis que la fausse coordination de l'attelage est plutôt une coordination hétérogène et la fausse attribution de l'hypallage une attribution biaisée.

Si l'on s'intéresse d'abord à l'interrogation rhétorique, force est de constater qu'elle est bien conforme à la construction syntaxique d'une phrase interrogative, mais que son accomplissement ne correspond pas à l'intention qu'elle affiche. Elle constitue à ce titre un trope illocutoire tel que le définit C. Kerbrat-Orecchioni (1980:194), puisque sa valeur dérivée (l'assertion) se substitue à sa valeur littérale (l'interrogation). Les prétentions énonciatives de l'interrogation rhétorique dépassant sa configuration syntaxique, on lui attribue alors la valeur illocutoire d'une affirmation déguisée en question. Or, le locuteur d'une interrogation ne feint pas de poser une question, il feint de douter de la réponse, ce qui n'est pas du tout la même chose. Ce déplacement de perspective autorise à traiter l'interrogation rhétorique non comme une fausse question mais comme une variante saillante de question dont l'ambigüité illocutoire consiste en «l'allocution d'un doute feint» (Bonhomme, 2012: 52) et en la reconnaissance par l'allocutaire d'une réponse détenue par le locuteur lui-même. La subjection peut elle aussi être reconsidérée dans les mêmes termes : elle ne feint pas le dialogue (il y a bien une séquence question-réponse) mais elle feint le doute, typique de l'attitude questionnante, tout en explicitant la réponse immédiatement après la question (Bonhomme, 2005b). On le voit, il est nécessaire de 
prendre en compte le contexte, en l'occurrence les conditions de production et de réception du discours, pour analyser les configurations potentiellement figurales.

Les cas de l'attelage et de l'hypallage semblent à priori moins récupérables puisque dans l'un et l'autre la configuration n'est cette fois-ci pas conforme aux modèles de la coordination et de l'attribution caractérisante. Le discours critique décrit alors la figure par la grammaire : dans l'hypallage, il y a une «modification des incidences syntaxiques " et "fausse attribution des adjectifs à ce qui n'est pas leur support "logique" " dans la phrase (Cotte, $1991: 80$ ), cette figure se rencontrant aussi bien dans le parler ordinaire ( place assise, rencontre amoureuse) que dans le discours littéraire (ex. 12). L'attelage, quant à lui, relie des éléments qui ne peuvent pas être coordonnés selon la logique grammaticale, logique qui n'autorise que la coordination de caractérisants homogènes et fonctionnellement équivalents : est accepté une robe rouge et qui se porte facilement mais pas *une robe royale et rouge (requalifié comme figure sous certaines conditions du discours). Dans les deux cas, rhétorique et grammaire se renvoient la responsabilité de décrire le fait. À moins que ce ne soient les figures qui renvoient grammaire et rhétorique à leurs obligations respectives : pour l'une, décrire un phénomène linguistique comme conforme ou contraire au système, ou encore simplement possible; pour l'autre, dégager « les motivations contextuelles » et "les effets tant illocutoires que perlocutoires » de ces mêmes phénomènes (Bonhomme, 2008 : 1491).

\section{2. ... ou la figure comme « variante saillante »?}

Pour M. Bonhomme (2005a: 31), les figures doivent être abordées « dans la perspective graduelle des linguistiques variationnelles » et par rapport aux « variations concurrentes qui interviennent dans la construction de chaque discours ». Parmi elles, on trouve les variations jugées standardisées, c'est-à-dire les constructions admises qui acquièrent «le statut de norme pour le français actuel, tel qu'il apparait dans les grammaires ou les dictionnaires " (ibid. : 32). À côté de ces variations standardisées, mais toujours relatives, le locuteur peut choisir des formes libres, davantage idiolectales, qui suscitent « différents marquages à l'intérieur des occurrences produites » et « instaurent un jeu contrastif avec les canevas institutionnalisés ou routiniers" (ibid.:33). On retrouve l'idée chère aux rhéteurs que la figure est «proche» de l'expression correcte. M. Bonhomme envisage trois types de variations libres: 1) les variations libres jugées recevables, qui correspondent à la syntaxe d'expressivité traditionnelle ; 2) les variations libres jugées non recevables, qui reçoivent un jugement d'agrammaticalité; enfin 3) les variations libres jugées exemplaires, dont le statut est « valorisé, sur le plan discursif, même s'il est souvent senti comme transgressif» (ibid.:33). L'interrogation rhétorique, comme variante libre exemplaire, est reconsidérée comme une variante saillante du phénomène linguistique de l'interrogation (Bonhomme 2012) et évènement discursif remarquable dans l'énoncé par son jeu sur les attendus traditionnels de l'acte de langage questionner.

\subsection{L'hypallage : de la fausse attribution à la variante saillante d'attribution}

31 L'hypallage promeut une configuration dans laquelle « la construction correcte s'oppose à la prédication impropre» (Rastier, 2001: 15), par une discordance entre structure syntaxique et structure sémantique, comme dans l'exemple 12. 

mauvaise distribution patente du caractérisant dans l'énoncé (incident à bruit là où l'attendu lexical le placerait plus volontiers à côté d'aviron) et, d'autre part, la caractérisation incongrue qui en résulte et provoque le calcul interprétatif de l'énonciataire. Manifestement le «bruit plat » constitue une surprise référentielle pour le lecteur. Dans l'univers décrit, le « plat » que perçoit le centre de perception (je) provient de l'aviron sur l'eau. Ce que perçoit l'oreille - dans cette atmosphère brumeuse qui brouille la vue - est la projection de la forme de l'aviron sur le son qu'il produit avec l'eau. Il est donc tout à fait probable que l'impression sur l'oreille "aplatisse " le bruit. L'hypallage exprime ainsi la perception synesthésique de l'énonciateur, qui ne reconduit pas une manière de dire conventionnelle ou attendue, ni un découpage notionnel consensuel, mais lui préfère une caractérisation ajustée à sa perception (Gaudin-Bordes \& Salvan, 2008). L'hypallage n'est donc pas tant une «fausse attribution » qu'une variante saillante d'attribution, à la fois libre et exemplaire par le relief qu'elle donne au discours, en l'occurrence par l'ajustement perceptif et énonciatif dont elle témoigne (GaudinBordes \& Salvan, 2012: 116). Elle réorganise linguistiquement l'expérience sensorielle et cognitive propre à l'énonciateur qui tente ainsi de représenter un réel perçu pour celui à qui il s'adresse (Gaudin-Bordes \& Salvan, 2014 ; Legallois, 2005). À cette attribution à effet figural s'opposeront toutes les attributions incongrues qui ne produisent pas de « relief exemplaire », et qui auront par conséquent moins de chance de passer la rampe du discours, et seront même versées dans les anomalies du discours.

L'hypallage peut donc être analysée comme une variation exemplaire, à la fois pertinente et pleinement fonctionnelle dans son contexte de production, même si elle n'est pas enregistrée par la norme. Elle tire sa figuralité non seulement de sa saillance, mais de son gain sémantique et interprétatif que sa contextualisation permet de préciser.

\section{Conclusion : d'une pensée de l'écart à l'hyperpertinence des figures}

L'étude des figures en contexte amène nécessairement à repenser la norme qui sous-tend les productions langagières, et à envisager plutôt les normes que la figure convoque ellemême: normes linguistiques, génériques, idiolectales ou textuelles. L'examen de quelques figures frappées régulièrement d'ostracisme montre que la figure est plus un «fait gênant» qu'un "fait contradictoire» pour la langue (Willens, 1985: 95) ou qu'un impossible de la langue. Parallèlement, loin de subvertir les normes langagières, elle permet parfois de pallier momentanément les lacunes de la langue, tout en la rénovant au moyen de formes qui engagent fortement les deux pôles de la communication. Les figures, qui relèvent plutôt de l'inédit que de la faute, peuvent être repensées comme pleinement pertinentes et fonctionnelles dans leur contexte de production et comme agissant en retour sur le contexte, ce dont le concept d'hyperpertinence rend compte (Gaudin-Bordes \& Salvan, 2010, 2013). Les figures n'existent que prises en charge dans la dynamique des interactions verbales et constituent des zones d'interaction rendant au mieux la complexité $\mathrm{du}$ réel. Ce processus figural est nécessairement graduel et la contextualisation des figures permet de mettre en évidence la variabilité du régime de la figuralité, c'est-à-dire son « instabilité » (Bonhomme, 2013) qu'une approche trop rigide perd de vue. Si faute il y a dans la figure, ce n'est donc que parce qu'elle s'affiche comme 
telle ; si elle est pardonnée, c'est qu'elle puise dans le discours ses conditions de félicité et d'interprétation.

\section{BIBLIOGRAPHIE}

Bonнomme, M. (2000). « Pour une rhétorique modulaire : réexamen de trois figures de construction canoniques ». In: Englebert, A. (éd.), Actes du XXII ${ }^{e}$ Congrès international de linguistique et philologie romane. Tübingen : Niemeyer, p. 9-14.

- (2014) [2005a]. Pragmatique des figures du discours. Paris : Champion.

- (2005b). «Flou et polyvalence de la question rhétorique ». In: Rossari, C. (dir.), Les États de la question. Québec : Nota Bene, p. 191-209.

- (2008). « Pour une intégration modulaire de la stylistique dans une linguistique de l'écrit ». In: Durand, J., Habert, B. \& Laks, B. (éds), Congrès mondial de linguistique française. Paris, Institut de linguistique française, p. 1491-1495.

- (2009). « Entre grammaire et rhétorique. L'hyperbate comme extraposition problématique ». In: Apothéloz, D., Combettes, B. \& Neveu, F. (dirs), Les Linguistiques du détachement. Berne : Peter Lang, p. 117-127.

- (2012). «Polyphonie et ethos dans l'interrogation rhétorique : le cas des Fables de La Fontaine ». In: Calas, F. et al. (dirs), Les Figures à l'épreuve du discours. Dialogisme et polyphonie. Paris : Presses de l'université Paris-Sorbonne, p. 39-52.

- (2013). «Vers une appropriation linguistique des figures ». L'Information grammaticale 137, p. 3-8.

- (2013). «La métaphore injurieuse comme construction contextuelle ». Le Discours et la langue 4.2 , p. 25-35.

COTTE, P. (1991). « L'hypallage ou la fausse attribution ». Théorie, littérature, enseignement 9 , p. 79-95.

DIDEROT, D. \& LE ROND D’ALEMBERT, J. (1751-1772). L'Encyclopédie ou Dictionnaire raisonné des sciences, des arts et des métiers.

DUPRIEZ, B. (1984). Gradus. Les procédés littéraires. Paris : Union générale d'édition.

FABRI, P. (1969) [1521]. Le Grand et Vrai Art de pleine rhétorique. Genève : Slatkine.

FONTANIER, P. (1977) [1821-1830]. Les Figures du discours. Paris : Flammarion.

FROMilhague, C. (1995). Les Figures de style. Paris, Nathan.

GARdes TAMine, J. (2011). Pour une nouvelle théorie des figures. Paris : Presses universitaires de France.

GAUDIN-BORDES, L. (2008). « La tyrannie tautologique : l'évidence comme outil énonciatif et stratégie discursive ». Langue française 160, p. 55-71.

GAUDIN-BORDES, L. \& SALVAN, G. (2008). « Le sens en marche : le cas de l'hypallage ». L'Information grammaticale 116, p. 15-19.

- (2009). «Figures du discours et frontières notionnelles ", Cahiers de praxématique 53, p. 121-142. - (2010) : « De la non-pertinence à l'hyperpertinence : intrig(u)antes figures dans Voyage au bout 
de la nuit ». In: Denis, D. et al. (dirs), Au Corps du texte. Hommage à Georges Molinié. Paris : Champion, p. 279-295.

- (2013). «Contextualisation et hyperpertinence figurale ». Le Discours et la langue 4.2, p. 17-24.

- (2014). «L'hypallage, un opérateur synesthésique? ». In: Verine, B. (éd.), Dire le non visuel:

approche pluridisciplinaire des discours sur les perceptions autres que la vue. Liège : Presses universitaires de Liège.

- (2016, à paraitre). « Le pléonasme : simplement redondant?». In : Bertocchi, S., Kurts, L., Paillet, A.-M. \& Stolz, C. (dirs), La Simplicité. Manifestations et enjeux culturels du simple en art. Paris : H. Champion.

GRICE, H. P. (1979). « Logique et conversation ». Communication 30, p. 57-72.

KERBRAT-ORECCHIONI, C. (1980). L'Énonciation. De la subjectivité dans le langage. Paris : Armand Colin.

LEGALLOIS, D. (2005). « Synesthésie adjectivale, sémantique et psychologie de la forme : la transposition au cœur du lexique ». In: François, J. (dir.), L'Adjectif en français et à travers les langues . Caen : Presses universitaires de Caen, p. 493-506.

maingueneau, D. (1990). Éléments de linguistique pour le texte littéraire. Paris : Bordas.

MELLET, S. (2012) [2009]. « La frontière notionnelle en langue et en discours ». Cahiers de praxématique 53, p. 7-20.

MOLINIÉ, G. (1986). Éléments de stylistique française. Paris : Presses universitaires de France.

- (1992). Dictionnaire de rhétorique. Paris : Librairie générale française.

QUINTILIEN (1978). Institution oratoire. Paris : Les Belles Lettres.

RASTIER, F. (2001). «Indécidable hypallage ». Langue française 129, p. 111-127.

RICALENS-POURCHOT, N. (2010). Lexique des figures de style. Paris : Armand Colin.

ROBRIEUX, J.-J. (2010). Rhétorique et argumentation. Paris : Armand Colin.

SALVAN, G. (2013). « Les figures de construction à la lumière de l'énonciation ». L'Information

grammaticale 137, p. 43-49.

- (coord.) (2013). « Figures du discours et contexte ». Le Discours et la langue 4.2.

SEGUIN, J.-P. (1999). « La langue française aux XVII ${ }^{\mathrm{e}}$ et XVIII ${ }^{\mathrm{e}}$ siècles ». In: Chaurand, J. (dir.), Nouvelle Histoire de la langue française. Paris : Seuil, p. 225-344.

Stolz, C. (2011). « Tout ce que vous avez toujours voulu savoir su l'hyperbate, sans jamais oser le demander ». In: Paillet, A.-M. \& Stolz, C. (dirs), L'Hyperbate, aux frontières de la phrase, Paris, Presses de l'université Paris-Sorbonne, p. 27-44.

VERINE, B. (2008). « La parole hyperbolique en interaction : une figuralité entre soi-même et même ». Langue française 160, p. 117-131.

WILLENS, D. (1985). « La problématique des données et la place de l'exception en syntaxe contemporaine ». Langue française 66, p. 86-98.

\section{NOTES}

1. Au sens très précis qu'en donne M. Bonhomme (2012:40) : «Relief qui détache toute figure sur l'arrière-plan du discours ".

2. En ligne : http://angely.over-blog.com/article-25738573.html (consulté le 27/03/13). 
3. On voit s'opérer une substitution de paradigme, des figures de redondance (redondance, périssologie et tautologie, mais aussi métabole, synonymie, paraphrase, battologie), aux caractérisations non pertinentes (alliance de mots, oxymore, hypallage et zeugme).

4. À l'inverse de la redondance qui opère une « soustraction » sémantique, comme l'apparition de à l'avance dans prévoir à l'avance tend à sortir le sème /anticipation/ de prévoir.

5. Voir l'étude de cet exemple dans L. Gaudin-Bordes \& G. Salvan (2009).

6. Néologisme créé par San Antonio, cité par N. Ricalens-Pourchot $(2010: 103)$.

7. Attracteur par ailleurs indicible «sauf sous la forme de tautologies: "laver plus blanc que blanc" ; “il était pâle, mais pâle !..."; “il y avait un de ces vents, j’te dis pas...”) » (Mellet, 2012 : 13).

8. Qui rassemble, mais pas plus, les caractéristiques de la notion.

9. Pour une étude du pléonasme dans une approche énonciative et pragmatique et son rapport au simple, voir L. Gaudin-Bordes \& G. Salvan (2016, à paraitre).

\section{RÉSUMÉS}

Les figures du discours sont souvent l'objet de discours grammaticaux normatifs et de jugements axiologiques qui opèrent comme des filtres: ce sont à ces jugements d'exclusion, et aux stratégies de réintégration dans l'analyse linguistique actuelle que s'intéresse cet article, en prenant deux figures comme illustrations, le pléonasme (figure « fautive ») et l'hypallage (figure «fausse »). L'étude de ces figures en contexte en permet une réévaluation positive, et argumente en faveur de la gradualité et de la variabilité de la figuralité, évaluée à la lumière de la notion d'« hyperpertinence ».

Figures of speech are often subjected to normative grammatical discourses and axiological judgements that act as filters. These judgements leading to exclusion, and the reintegration strategies of current linguistic analyses are discussed in this article, and illustrated by pleonasm (an « offending » figure) and hypallage (a «false» figure). Studying figures in their contexts allows us to reevaluate them (in a positive light) and substantiates the notions that figurality is variable and gradual, and can be evaluated using the concept of "hyperpertinence".

\section{INDEX}

Keywords : figures of speech, pleonasm, hypallage, normativity, hyperpertinence

Mots-clés : figures du discours, pléonasme, hypallage, normativité, hyperpertinence

\section{AUTEUR}

\section{GENEVIÈVE SALVAN}

BCL (UMR CNRS 7320), Université Nice Sophia Antipolis 